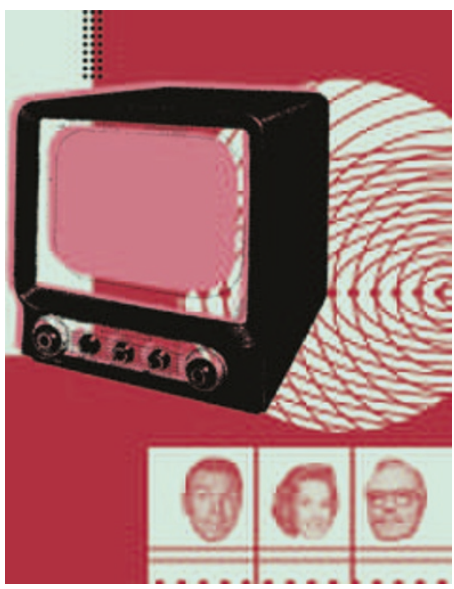

RESEARCH HIGHLIGHTS

URLs

\title{
A screen success
}

Screening human cell lines for genes involved in cancer can be tricky - such genes are often recessive and the mechanisms through which they are inactivated can be complex. RNAi screens provide a potentially powerful way to identify genes that are involved in cancer by investigating the effects of reduced gene expression on specific cellular phenotypes. Now, two independent groups have verified the importance of RNAi-based screening in human cell lines by using this approach to identify novel tumour-suppressor candidates.

Both groups produced libraries of retroviral vectors that contain short hairpin RNAs (shRNAs); each construct was complementary to a single human gene and 8,000 or so genes were represented in total. The researchers transduced these vectors en masse into engineered human cells lines that were poised for transformation - the rationale being that knocking down a candidate tumour suppressor would be enough to tip these cells over into the transformed phenotype.

Because most cancers arise from epithelial tissues, Elledge and colleagues chose an immortalized mammary epithelial cell line for their screen. For normal growth in vitro, these cells need support from the extracellular matrix, but when they are transformed, they become 'anchorage-independent' and can simply be screened for proliferative ability on soft agar. Elledge's group also took the opportunity to carry out the first test of the use of 'genetic barcodes' in mammalian cells. By adding a unique 60 -nucleotide barcode to each shRNA vector, they used microarrays to successfully identify the shRNA responsible for the knockdown.

Reassuringly, several of the genes that Elledge and colleagues picked up are well-known tumour suppressors, such as transforming growth factor- $\beta$ receptor II (TGFBR2) and phosphatase and tensin homologue (PTEN). But they also found a new candidate tumour-suppressor gene - RE1-silencing transcription factor (REST) - which (like TGFBR2) resides in a locus that often suffers loss of heterozygosity, a frequent chromosomal characteristic of human tumour suppressors.

REST is often downregulated in prostate and small-cell lung cancers, and is frequently deleted in colon tumours. The authors present evidence that REST downregulation augments phosphatidylinositol 3-kinase signalling, an important pathway in cancer progression. Furthermore, the fact that REST is responsible for repressing neuronal genes in non-neuronal tissues provides another clue to its potential function in cancer. Several human tumours aberrantly express neuronspecific genes, and the authors reasoned that this might have an important role in cancer, with REST potentially playing a part.

Agami's group introduced their shRNA vectors into an immortalized fibroblast cell line that required only the deregulation of RAS for transfor- mation. RAS proteins convey growth signals from the cell surface to the nucleus, and their overexpression or aberrant activation is an important cause of cancer. Although activating mutations of $R A S$ are frequent in human cancers, many tumours still retain wild-type copies of the gene, prompting Agami and colleagues to look for genes that, when inhibited, activate RAS.

They identified the gene pairedlike homeodomain transcription factor 1 (PITX1), which is frequently downregulated in bladder and prostate tumours. Although it is not yet known whether PITX1 is mutated or deleted in human cancers, the authors present some intriguing evidence that loss of PITX1 can lead to RAS activation and a transformed phenotype.

Although REST and PITX1 are exciting candidates, further studies will be needed before their roles in cancer can be confirmed. Nevertheless, these studies show how powerful screens using RNAi libraries can be for identifying potential tumour-suppressor genes.

Jenny Bangham

(2) References and links ORIGINAL RESEARCH PAPERS Westbrook, T. F. et al. A genetic screen for candidate tumor suppressors identifies REST. Cell 121, 837-848 (2005) | Kolfschoten, I. G. M. et al. A genetic screen identifies PITX1 as a suppressor of RAS activity and tumorigenicity. Cell 121, 849-858 (2005) 